\title{
Les conditions géologiques et les traces de la bataille de Verdun
}

Jean-Claude Porchier, Dominique Harmand et Jacques Le Roux

\section{OpenEdition}

\section{Journals}

Édition électronique

URL : https://journals.openedition.org/geohist/578

DOI : 10.4000/geohist.578

ISSN : 2264-2617

Éditeur

Association française de la Revue de géographie historique

\section{Référence électronique}

Jean-Claude Porchier, Dominique Harmand et Jacques Le Roux, « Les conditions géologiques et les traces de la bataille de Verdun », Revue de géographie historique [En ligne], 14-15 | 2019, mis en ligne le 28 mai 2018, consulté le 12 juin 2021. URL : http://journals.openedition.org/geohist/578 ; DOI : https://doi.org/10.4000/geohist.578

Ce document a été généré automatiquement le 12 juin 2021.

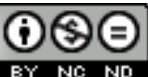

Ce(tte) œuvre est mise à disposition selon les termes de la Licence Creative Commons Attribution Pas d'Utilisation Commerciale - Pas de Modification 4.0 International. 


\section{Les conditions géologiques et les traces de la bataille de Verdun}

Jean-Claude Porchier, Dominique Harmand et Jacques Le Roux

\section{Cadre géologique et atouts défensifs}

1 Verdun se trouve au cœur du relief de côtes de la Lorraine sédimentaire, àl'est des côtes de l'Argonne(ou côte de l'Albien) et des Bars (ou côte du Tithonien), dans la vallée de la Meuse, encaissée dans le revers de la côte de Meuse (ou côtede l'Oxfordien) (Fig. 1). Le revers de la côte à l'est de la Meuse constitue sur sa rive droite une tête de pont d'importance stratégique majeure par la protection qu'elle offre à la ville. Les Allemands, échaudés par leur défaite sur la côte de Moselle et les buttes-témoins du Grand Couronné de Nancy, ne se risquèrent pas à l'attaquer en partant de la plaine marécageuse de la Woëvre, impropre aux manœuvres militaires. 
Document 1 : Carte géologique du site de la bataille de Verdun (Harmand D., 2018)
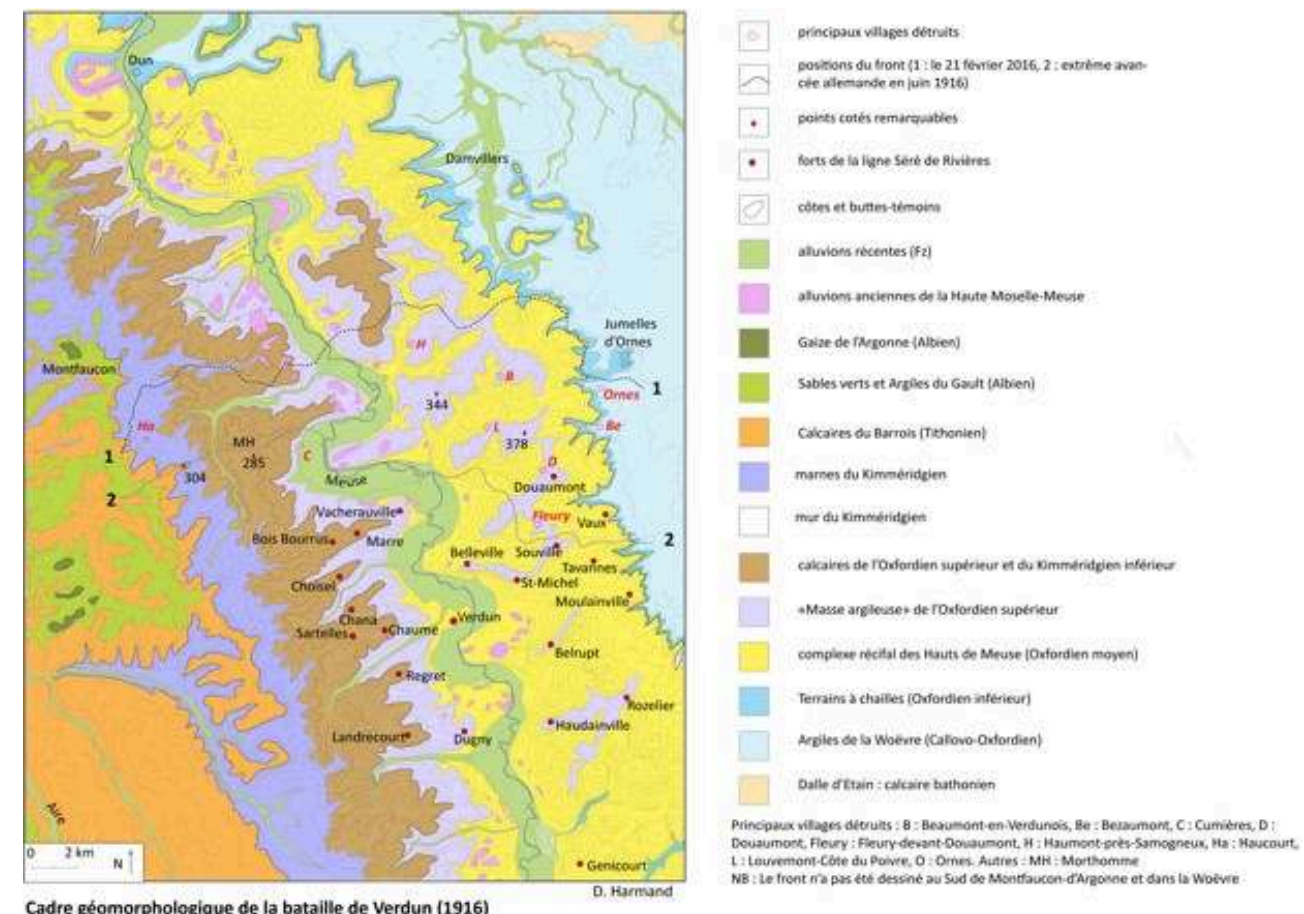

Les ondulations anticlinales et synclinales d'orientation WSW-ENE qui affectent les deux rives de la Meuse se manifestent par des crêtes bordées de ravins qui furent autant d'obstacles à la progression allemande. Ces ondulations se manifestent également par des perturbations des fronts de côte sous forme de rentrants et de saillants. En avant de ces fronts, les buttes-témoins ont offert des observatoires exceptionnels et ont été âprement disputées. S'il était difficile de progresser depuis le nord en direction de Verdun à cause de ces ondulations qui constituaient autant de points de résistance et de dissimulation de l'artillerie à contre-pente, il était également difficile de progresser vers le sud par la vallée de la Meuse. En effet, celle-ci n'est pas la voie de communication que l'on pourrait penser. Ses méandres encaissés empêchaient l'assaillant d'avoir une vision lointaine, ses flancs couverts de cultures permanentes, surtout prés-vergers et un peu de vigne, n'offraient aucun couvert, et son lit marécageux devenait impraticable à la moindre inondation.

\section{La défense de Verdun}

Dans le cadre du système Séré de Rivières, la place forte de Verdun était l'extrémité nord du rideau défensif des Hauts de Meuse aménagé entre Verdun et Toul. Au sud, entre les deux places fortes de Toul et Verdun, le rideau des Hauts de Meuse comprenait les ouvrages suivants : au nord de la trouée de Spada, le fort de Génicourt et le fort de Troyon, au sud, les forts des Paroches, du Camp-des-Romains et de Liouville, la batterie de Saint-Agnant et les forts de Gironville et de Jouy-sous-les-Côtes. Le fort de Troyon et le fort des Paroches étaient séparés par la trouée de Spada que le second était conçu pour bloquer en déversant un tir d'enfilade sur toute force approchante. La place forte de Toul était défendue au sud par le fort du Pont Saint-Vincent, dernier ouvrage avant la Trouée de Charmes, qui était suivie du rideau de la Haute-Moselle, d'Épinal à Belfort. 

renforcement du front et àla création de positionssuccessives en arrière (Fig. 2), et en février 1916, dans le secteur d'attaque des Allemands, la défense était échelonnée sur quatre positions (Solard, 1935).

Document 2 : Schéma des positions de défense de Verdun au 21 février 1916 (Solard, 1935)

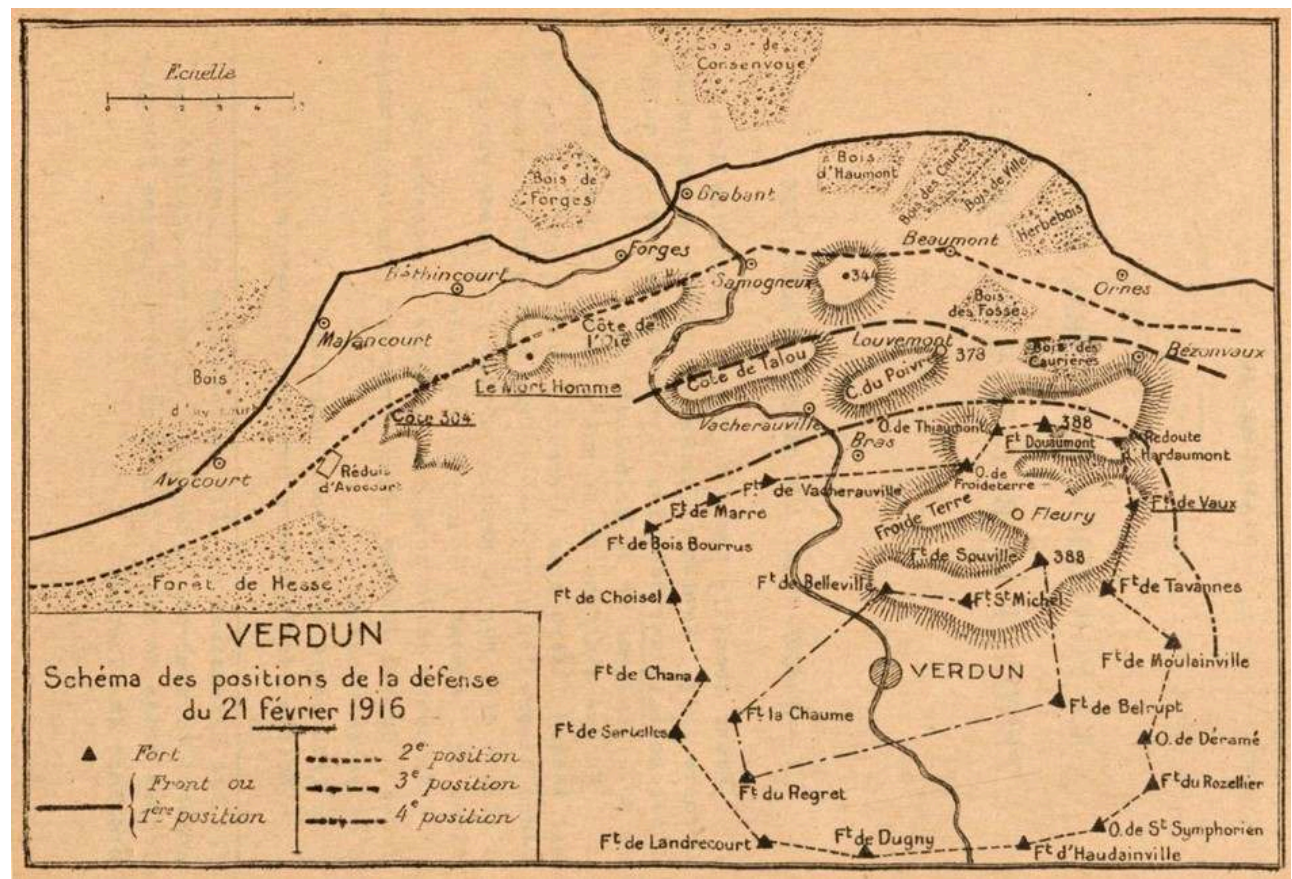

mière s'étendait d'Avocourt à Ornes.Elle illustrait la militarisation de la forêt, devenue de 1873 à 1914 " acteur de la défense du territoire » (Amat, 2015) et s'appuyait sur une rangée de bois : bois d'Avocourt, de Forges, d'Haumont, des Caures, de Ville et Herbebois. Les bois profitaient aussi aux Allemands qui préparaient leur attaque dans les forêts plus au nord, notamment Consenvoye, et Spincourt (Fig. 2). Bien que considérée comme suffisante elle manquait d'abris de bombardement et de défenses accessoires. L'existence de centres de résistance isolés et visibles devait faciliter leur écrasement par l'artillerie ennemie.

7 Les deuxième, troisième et quatrième positions, avaient été commencées fin 1914, mais les travaux n'avaient pas été achevés du fait du manque de personnel, et Solard jugeait que ces organisations n'avaient qu'une faible valeur sauf la quatrième, mieux entretenue.

8 La deuxième position s'appuyait sur un puissant système défensif naturel comprenant d'ouest en est, la cote 304, éperon de la côte des Bars (Tithonien), le Mort-Homme et la côte de l'Oie (Oxfordien supérieur et Kimméridgien), protégés au nord par la vallée du ruisseau de Forges, et se prolongeant sur la rive droite par la cote 344 sur un lambeau de la « masse argileuse de l'Oxfordien supérieur », désignée comme « Séquanien » dans les cartes anciennes.

La troisième position, sur la rive droite, s'appuyait sur la côte de Talou et la côte du Poivre, sur le « Séquanien ». 
10 La quatrième position était la seule à porter des fortifications permanentes. La ligne Bois Bourrus, Marre, Vacherauville (sur un éperon d'Oxfordien supérieur et Kimméridgien comparable au Mort- Homme), prolongée par la ligne Froideterre, Douaumont, Vaux, Moulainville (sur une arête de "Séquanien ») formait une position extrêmement puissante. Toutefois, suite à la " crise de l'obus-torpille », en 1914, on ne croyait plus à l'efficacité des fortifications. Au lieu d'incorporer ces forts dans les lignes pour leur servir de points d'appui, on décida de les démanteler et de les faire sauter pour ne pas les laisser tomber entre les mains de 1'ennemi. Les garnisons et les approvisionnements furent supprimés, les casemates de Bourges furent désarmées et on ne laissa que les canons sous tourelles qui ne pouvaient être récupérés par l'artillerie de campagne. La tourelle centrale du fort de Vaux, détruite par un obus ennemi qui fit exploser les charges destinées à sa destruction (Fig. 3), témoigne de cette période d'hésitation, qui se termina quand le général Pétain prit la direction de la bataille et rendit les forts à leur destination primitive d'intégration à la ligne de combat.

Document 3 : La tourelle détruite du fort de Vaux (cl. J.-C. Porchier)

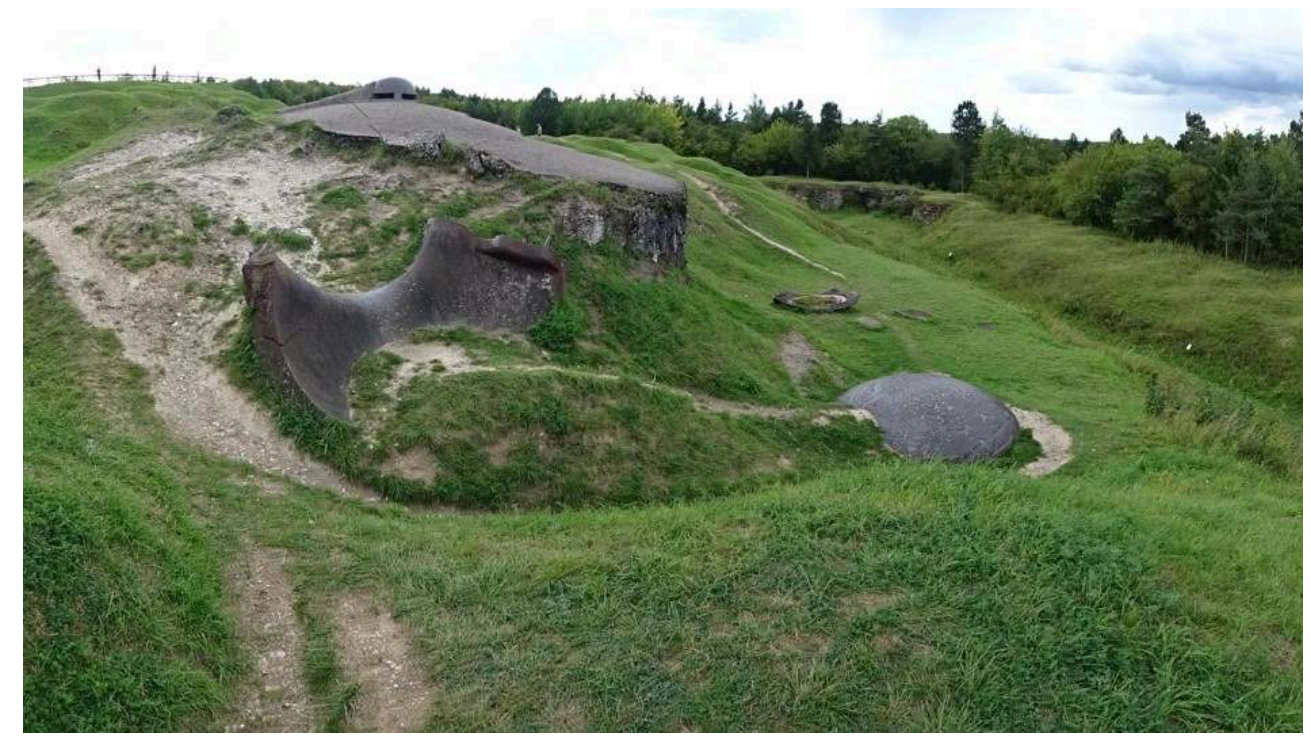

11 Les forts les plus à l'est, Vaux et Moulainville, n'étaient plus sur le « Séquanien », mais sur les terrains plus anciens du complexe récifal des Hauts de Meuse (Oxfordien moyen, «Rauracien» des auteurs). Ils n'étaient pas alimentés comme les autres forts et les villages par la nappe perchée de la base du « Séquanien », ce qui fut une des raisons de la chute du fort de Vaux qui se procurait son eau au ravin des Fontaines et ce qui fut impossible une fois le fort assiégé.

Les forts situés plus au sud : fort de Choisel, ouvrage du Chana, fort de la Chaume, fort des Sartelles, fort de Regret, forts de Landrecourt et Dugny, trop éloignés du front, n'ont pas été attaqués et ont servis de bases arrière. Plus au sud, le fort de Génicourt faisait la liaison avec le rideau des Hauts de Meuse. 


\section{L'influence de la géologie sur les combats}

13 Les Allemands, déjà tenus en échec sur la côte de Moselle à Nancy, se gardèrent d'attaquer depuis la Woëvre, dont le sol argileux était impropre aux manœuvres et où le petit nombre de voies praticables forçait à une concentration des troupes qui les rendait vulnérables à l'artillerie placée sur les Hauts de Meuse. Ils décidèrent de ne pas attaquer le front de côte mais de le prendre de flanc en attaquant sur la rive droite par le nord, avec une concentration d'artillerie sans précédent, dont notamment les fameux obusiers de 420 surnommés « Grosse Bertha ». Mais ce qui avait été efficace sur les forts de Belgique le fut moins sur les obstacles naturels auxquels se cramponnaient les Français. En effet, sur la rive droite, entre la vallée de la Meuse et la côte de Meuse, les calcaires de l'Oxfordien moyen et supérieur qui forment le revers de côtedes Hauts de Meuse, sont surmontés par un réseau d'arêtes SW-NE, sortes de buttes-témoins des côtes de Meuse occidentales posées sur la "Masse inférieure argileuse ", formant talus et reliées entre elles par des crêtes NW-SE. Comme la possession de ces hauteurs donnait un avantage tactique, elles donnèrent lieu aux combats les plus acharnés et, comme le fait remarquer Johnson, les accidents naturels du relief, escarpements, ravins, zones inondées s'avérèrent plus efficaces que des fortifications bâties. En outre, l'artillerie des forts de Bois Bourrus, Marre et Vacherauville sur la rive gauche, joua un rôle majeur pour contrôler les attaques sur la rive droite. A l'inverse, les obusiers de 420 allemands perdirent rapidement de leur efficacité du fait de la difficulté à les déplacer sur un terrain accidenté et détrempé.

14 Tenus en échec sur la rive droite, les Allemands furent bientôt contraints d'élargir le front d'attaque à la rive gauche malgré les inconvénients causés par la présence en son milieu de la vallée de la Meuse qui, gonflée par des crues d'hiver, avait séparé les deux ailes de l'offensive et empêchait leur coordination.

15 À la fin juin, le Kronprinzfut près de s'emparer de la quatrième position sur l'arête Froideterre, Douaumont, Vaux, Moulainville, mais il échoua. Dès le 4 juin, l'armée russe avait été engagée dans l'offensive Broussilov, et en juillet 1916 les Britanniques lancèrent l'offensive de la Somme et la tempête à Verdun s'apaisa. L'état-major allemand fut contraint d'alléger la pression sur Verdun et dut donner de nouvelles orientations à sa stratégie. L'effort de l'Allemagne contre le formidable obstacle des Hauts de Meuse avait échoué. Cependant, la récupération du terrain s'avéra difficile en raison du labyrinthe de trous d'obus remplis de boue et d'eau, et une grande partie du territoire gagné par les Allemands resta entre leurs mains. Mais Verdun était sauvée et demeurait un bastion imprenable.

Les différents faciès du Jurassique, et particulièrement de l'Oxfordien, avec ses formations récifales et péri-récifales hétérogènes, parfois massives et impossibles à creuser avec les outils de base du soldat ou fracturées et exigeant de gros travaux d'étayage n'étaient pas favorables àla construction d'ouvrages de terrain et au creusement de tranchées. Par endroits, et notamment sur la rive gauche (Cote 304 et Mort-Homme), le front était devenu à peu près partout un réseau de trous d'obus reliés par des boyaux sommairement creusés. L'acharnement des combats et l'absence de stabilisation du front avaient empêché la construction d'ouvrages défensifs bétonnés et la conduite d'une guerre de mines. Celle-ci est restée limitée aux extrémités du champ de bataille, pour la conquête de deux postes d'observation d'importance majeure, Vauquois au nord et Les Éparges au sud. Les Allemands eurent toutefois le temps de 
creuser trois tunnels pour faciliter les mouvements de troupes dans les marnes et calcaires rocailleux du Kimméridgien du Mort-Homme, qu'ils durent fortement étayer et dont il ne reste rien.

Les tranchées et les boyaux ont pour la plupart été très altérés par les bombardements et ne sont souvent plus visibles, ou du moins ne sont plus dans leur aspect initial. Le peu que l'on peut voir actuellement a probablement été recreusé après 1916, comme le boyau de Londres. Quelques vestiges sont visibles en périphérie : bois d'Ormont, poste de commandement du colonel Driant, cote 344... Leur étude est facilitée par l'emploi du lidar qui a permis de dresser une cartographie numérique détaillée et en trois dimensions du modelé du champ de bataille, révélant des structures masquées sous le voile végétal et offrant des perspectives renouvelées aux archéologues de la Grande Guerre (De Matos Machado et al, 2016).

\section{L'évolution de la forêt}

18 Avant même la Grande Guerre, la forêt était une composante de l'espace frontalier (Amat, 2015). L'Argonne fut à plusieurs reprises dans l'histoire de la France appelée les « Thermopyles françaises » (Pranard, 1842, avant le Nouveau Larousse illustré de 1925 habituellement cité comme source).

19 Toutefois, la qualité de la forêt actuelle de Verdun ne doit pas faire oublier qu'en 1914, le territoire du champ de bataille de Verdun était faiblement forestier, le taux de boisement ne dépassant pas les 12 \% (Académie de Nancy-Metz, 2016). Le paysage agraire et forestier avait presque entièrement disparu en 1918, et le paysage actuel est complètement différent.

20 Près de 90 ans après la bataille, la structure de la forêt domaniale de Verdun garde encore nettement la trace de son passé. Les trois grands sous-ensembles - peuplements résineux, peuplements feuillus adultes et végétation pré-forestière, jeunes peuplements - imbriqués les uns dans les autres en mosaïque, sont des témoins de l'histoire de ce territoire. Les peuplements résineux et les plantations marquent les emplacements des anciens terrains agricoles, alors que les bois feuillus adultes sont encore limités à leur présence effective au début du XXe siècle (ONF, 2005).

21 Des parcelles de forêt ancienne : poste de commandement du colonel Driant, Jumelles d'Ornes et Bois d'Ormont notamment contiennent un certain nombre d'arbres-témoins. De nombreux hêtres et quelques chênes des anciens bois d'avant-guerre sont ainsi remarquables par le simple fait qu'ils ont survécu à la bataille. On les reconnaît, sans toujours avoir de certitude, à leur diamètre important pour la forêt et leur port au houppier imposant (du fait de leur croissance sans concurrence). Certains portent encore les stigmates de la guerre, comme des barbelés ou certaines blessures encore visibles (ONF, 2005). En 1928, l'État a exproprié la plus grande partie de la zone rouge meusienne pour y créer de nouvelles forêts domaniales, dont celles du Mort-Homme et de Verdun. Le périmètre de celle-ci inclut 300 ha d'anciens bois communaux et privés et 6000 ha des terroirs agricoles de sept villages disparus (Amat, 2015).

Les essences choisies au lendemain de la guerre pour le reboisement (pin noir d'Autriche et pin sylvestre sur les sols secs, épicéa sur les sols argileux) impliquent que la forêt domaniale soit constituée d'une mosaïque de peuplements et de types de végétation. Ces essences étaient alors les plus susceptibles de s'acclimater rapidement 
sur les terrains dévastés et appauvris de la zone des combats et présentaient également l'intérêt d'empêcher la pousse d'un sous-bois touffu qui masquerait les vestiges Les pins d'Autriche ont été plantés principalement dans les zones très visitées, notamment en raison de l'éclairage doux qu'ils donnaient aux lieux de mémoire (Hupy et Koehler, 2012).

Toutefois, ces plantations, si elles ont permis la reconstitution rapide des sols, se sont révélées peu adaptées au milieu. Les peuplements résineux ont été peu à peu remplacés à partir de 1973, par des feuillus, hêtre notamment, qui correspondent mieux aux conditions écologiques de la région (ONF, 2005). Après les controverses de l'aprèsguerre sur l'opportunité de boiser ou de laisser le champ de bataille en l'état, une nouvelle controverse se fait jour, certains craignant que la suppression des résineux fasse disparaître ce paysage composite qui garde la mémoire des combats. La structure actuelle du paysage (peuplements résineux, plantations récentes, bois feuillus) de la forêt domaniale de Verdun est encore très nettement marquée par son histoire et différencie nettement ce territoire au sein des côtes de Meuse. La disparition à terme des résineux enlèverait une partie de sa spécificité à la forêt et contribuera à banaliser ce territoire unique.

\section{L'évolution du paysage agraire}

Si l'évolution du paysage forestier est bien documentée grâce aux archives des Eaux et Forêts, puis de l'ONF, celle du paysage agraire a donné lieu à moins de publications. Dans ce domaine, l'impact de la guerre n'est pas indiqué par des traces, mais plutôt par ce qui ne se voit pas, à savoir les terres agricoles disparues. Les huit villages rasés : Beaumont, Bezonvaux, Douaumont, Fleury, Haumont, Louvemont, Ornes et Vaux (Fig. 4 et 5) n'ont pas perdu que des bâtiments, ils ont aussi perdu des terroirs agricoles.

Document 4 : Paysage d'openfield autour de Vaux-devant-Damloup avant la guerre (carte postale, Chol édit., Verdun)

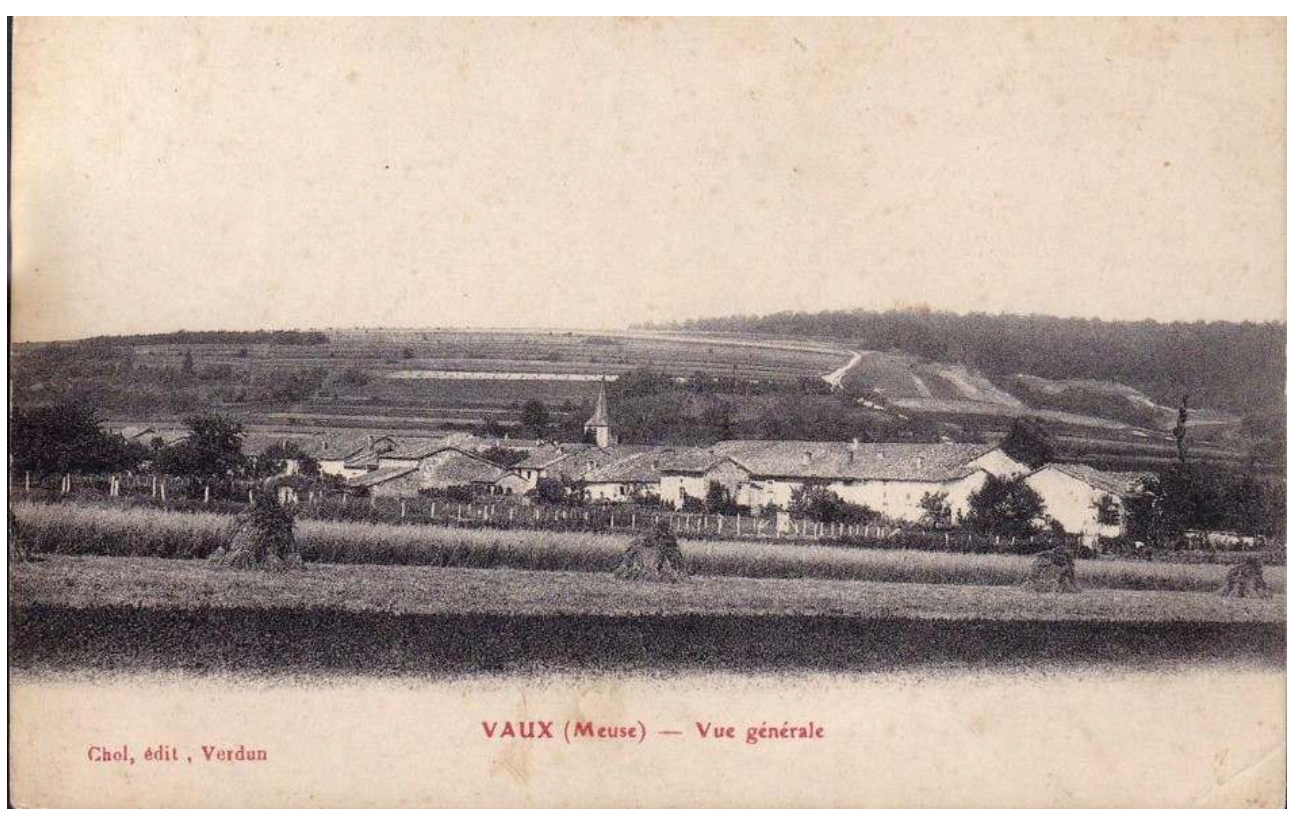


Document 5 : Le sommet de la cote 304 après l'attaque du 24 août 1917. 27 août 1917 (Collections La Contemporaine, cote VAL 185/162). Exemple extrême de bombturbation qui a réduit la hauteur de la crête de $6 \mathrm{~m}$.

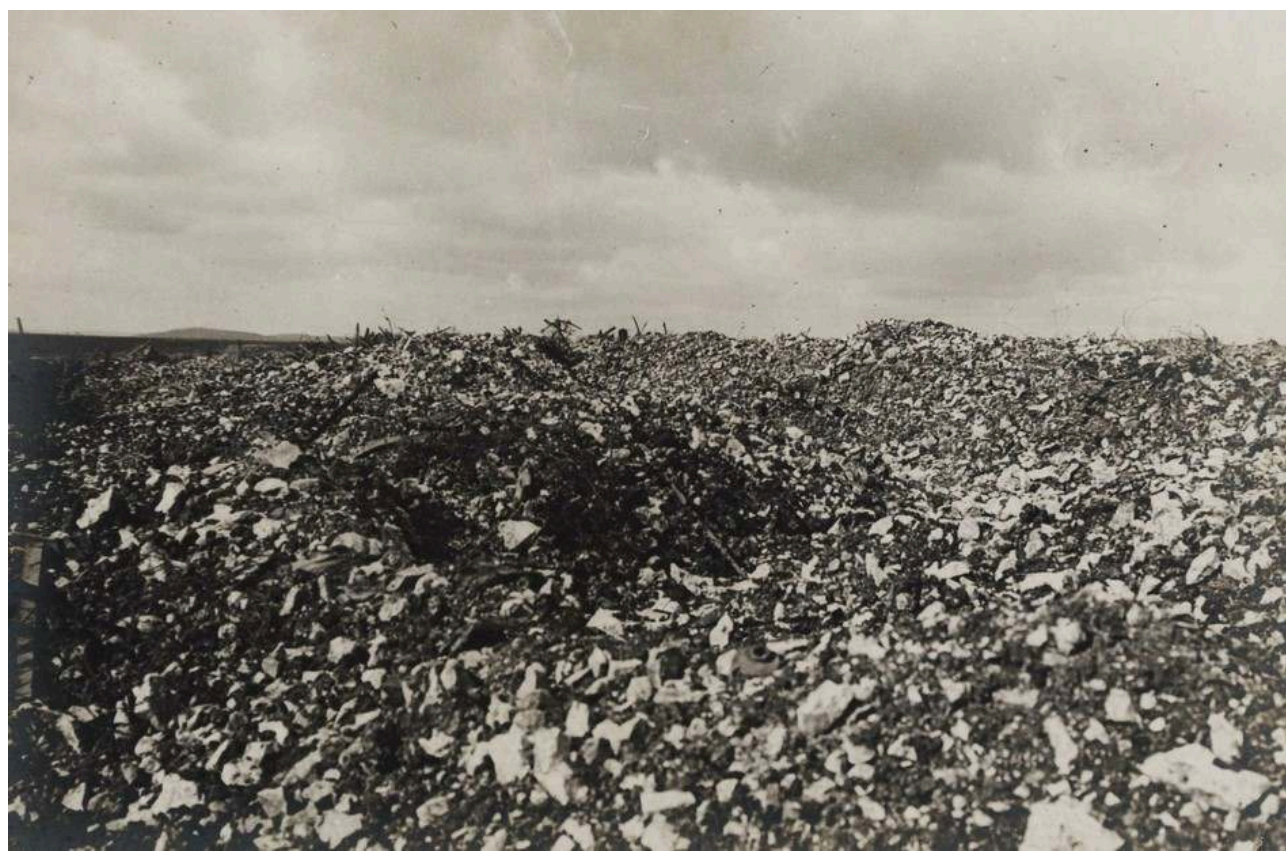

A la fin du XIX ${ }^{e}$ siècle, le paysage agraire de la Meuse était celui d'un openfield, avec les traits caractéristiques d'un découpage de parcelles en lanières d'une superficie voisine de l'unité traditionnelle du jour (journée dans d'autres régions), et de l'habitat groupé (villages-rues). Selon la monographie de Prudhomme (1893), la Meuse comptait en 1882 2,7 millions de parcelles d'une surface moyenne de 21 ares (bois compris).

a comparaison des données de différentes années d'avant-guerre compilées par Prudhomme en 1893 avec les premières données de l'après-guerre, publiées dans l'Enquête agricole de 1929 (Ministère de l'Agriculture, 1936) et à plus forte raison avec les données actuelles est hasardeuse compte tenu de la différence de méthodes et de nomenclature, mais la comparaison des deux sources permet d'illustrer l'impact du conflit sur les paysages agraires et forestiers.

En 1882, la Meuse était un département de petites exploitations, avec 56000 exploitations dans leur grande majorité de taille inférieure à 10 ha (Tab. 1). En 1929, la plupart des petites exploitations n'avaient pas survécu à la guerre et avaient disparu.

Tab. 1 : Evolution du nombre d'exploitations agricoles de la Meuse par taille entre 1882 et 1929.

\begin{tabular}{|l|r|r|}
\hline Taille & \multicolumn{1}{|c|}{1882} & \multicolumn{1}{c|}{1929} \\
\hline Moins de 1 ha & 20424 & 1280 \\
\hline De 1 ha à 10 ha & 26414 & 1971 \\
\hline De 10 à 40 ha (50 en 1929) & 8456 & 8277 \\
\hline Au dessus de 40 ha (50 en 1929) & 978 & 859 \\
\hline Au dessus de 100 ha (1929) & 56272 & 12645 \\
\hline Nombre total & & 258 \\
\hline
\end{tabular}

Les données de 1890 (Tab. 2) sur l'occupation des terres agricoles montrent une structure d'assolement triennal typique d'un openfield, avec des superficies à peu près 
équivalentes de blé (100 000 ha), de céréales secondaires (107 000 ha, majoritairement avoine), et de jachères et prairies artificielles de légumineuses (respectivement 57000 et 30000 ha). Les prairies naturelles occupaient 85000 ha.

Tab. 2 : Evolution de l'occupation du territoire de la Meuse de 1882 à 1929 (en hectares).

\begin{tabular}{|l|r|r|}
\hline & \multicolumn{1}{|c|}{$\mathbf{1 8 8 2}$} & \multicolumn{1}{c|}{1929} \\
\hline Terres labourables & 306375 & 207763 \\
\hline Vignes & 10802 & 272 \\
\hline Vergers et potagers & n.d. & 4862 \\
\hline Prairie & 85107 & 105503 \\
\hline Total SAU & 402284 & 318400 \\
\hline Friches et landes & 14786 & 74396 \\
\hline Forêt & 182112 & 207087 \\
\hline Autres & 23624 & 24174 \\
\hline & 622806 & 624057 \\
\hline
\end{tabular}
les dévastations n'ont été si complètes et si irréparables que dans la région des Côtes de Meuse et plus encore dans celle au Nord de Verdun. Des bois il ne reste plus un arbre intact : il faudra procéder un recépage général et planter des résineux qui serviront de couvert pour la régénération des anciens taillis avant de rétablir les chênes et les hêtres qui peuplaient les forêts en 1914 : c'est une oeuvre de très longue haleine Quant aux cultures, leur renaissance d'abord rapide (en 1920 dans le département de la Meuse 27000 ha étaient prêts à la culture mais 35000 restaient à niveler) a été fort retardée par la suppression des services d'État : les énormes travaux indispensables paraissent à peu près impraticables pour des particuliers. Près de 20000 ha resteront définitivement zone rouge : on pourra seulement créer sur quelques points bien choisis quelques îlots cultivables d'une étendue suffisante avec des bâtiments d'exploitation; chaque îlot sera entouré de maigres terres de parcours qui ne pourront être que des pâturages à moutons ; le surplus sera abandonné au boisement ».

Musset mentionne également le fort exode rural commencé dès avant la guerre où " la population rurale diminuait chaque jour davantage, enlevée aux champs par les mines et les usines, de nombreuses terres étaient délaissées » d'autant plus que l'on ne pouvait plus recourir comme avant à la main-d'œuvre polonaise par suite de la guerre entre la Pologne et la Russie soviétique de 1919-1921.

Revue de géographie historique, 14-15 | 2019 
33 A la question de la main-d'oeuvre s'ajoutait celle d'un extrême morcellement de la propriété foncière dans la région de Verdun qui faisait qu'on ne pouvait pas reconstituer les exploitations antérieures et qu'il fallait procéder abord à un remembrement, " opération particulièrement difficile vu l'état du sol » (Musset, 1922).

Si la superficie des terres labourables a fortement diminué après la guerre, l'enquête de 1929 ne montre pas de changement radical dans la nature des cultures. Elles sont toujours dominées par le blé et par l'avoine, nécessaires à l'alimentation des chevaux (on ne recense alors dans la Meuse que 75 tracteurs et 171 véhicules utilitaires). Le colza et le maïs sont pratiquement absents.

Les changements qui ont affecté l'agriculture après la Deuxième Guerre mondiale font qu'on ne retrouve plus trace du paysage d'openfield qui était encore visible dans les années 50, là où il n'avait pas été détruit par la Grande Guerre. En 2010, on ne comptait plus que 3000 exploitations. Les cultures étaient dominées par le blé tendre (71 800 ha), et les orges d'hiver et printemps (66000 ha). L'avoine a pratiquement disparu, remplacée par l'orge, et on a vu apparaître le colza (43800 ha, sur 47600 ha d'oléagineux), dont les fleurs jaunes sont fréquentes sur les photos actuelles du champ de bataille, mais qui était inexistant avant la Grande Guerre (231 ha en 1890).

La Meuse fut un des premiers départements où il fut procédé àun remembrement. Il le fut plus par nécessité, le trop petit parcellaire étant devenu incultivable, que par la volonté de mettre en place un système de production moderne et productiviste. Il servit en quelque sorte de modèle aux travaux préalables qui aboutirent à la loi de mars 1941 prise par l'État français de Vichy. Mais c'est après la Seconde Guerre mondiale que les procédures $\mathrm{du}$ remembrement se généralisèrent dans un grand nombre de communes, à cause de l'évolution rapide des structures d'exploitation, le nombre de celles-ci allant en diminuant rapidement depuis la fin du conflit et aussi des méthodes de culture modernes qui requièrent des parcelles de grande taille. D'autres circonstances, comme l'essor de l'économie des "Trente Glorieuses » ont renforcé ce besoin de remodelage parcellaire (Cabouret, 1989), et il ne reste presque plus de traces d'un parcellaire qui était encore visible entre les deux guerres.

En définitive, le grand changement paysager réside dans la perte définitive de $15 \%$ des surfaces agricoles de la Meuse, majoritairement dans l'arrondissement de Verdun, tombées en friche, classées en Zone Rouge et définitivement impropres à l'agriculture. Ces surfaces ont été reboisées, portant la superficie en forêts et surfaces boisées de $29 \%$ à $38 \%$ du territoire départemental, alors que les surfaces agricoles tombaient de $65 \%$ à $55 \%$ du territoire départemental.

Les destructions qui ont affecté les terres cultivables ont aussi affecté les villages. Ceuxci formaient un habitat groupé typique des terroirs d'openfield, sous la forme bien connue du village-rue lorrain avec son pittoresque tas de fumier sur l'usoir (espace entre les bâtiments et la chaussée) devant la ferme, signe extérieur de richesse aboli en 1980. Dès 1914, la loi a établi le droit individuel à une réparation des dommages subis par faits de guerre, dont les modalitésontétédéfinies par la loi du 17 avril 1919. Tout sinistré recevait une somme égale à la perte subie, estimée sur la base de la valeur du bien en août 1914. Afin d'encourager la reconstruction du patrimoine, la somme n'étaitperçue dans son entier que si elle étaitaffectée à un bien de même nature, ce qui a poussé à un certain conservatisme. 
39 L'habitat étant groupé, on a dessiné entièrement les villages, sous l'autorité du Génie Rural qui a établi des plans d'aménagement d'ensemble, avec l'aide de quelques architectes urbanistes. Les plans s'inspiraient des théories des cités-jardins qui ont émergé à la fin du XIX ${ }^{e}$ siècle en Angleterre dans une mouvance hygiéniste, et préparaient à une agriculture plus rationnelle et plus moderne, mais on retrouve la structure du village et de la ferme lorraine. De nombreuses fermes témoignent, plus modestement que les églises et bâtiments officiels, de cette reconstruction d'aprèsguerre. La brique est le matériau privilégié de la reconstruction, elle peut être de terre cuite, naturelle ou vernissée pour la décoration, ou fabriquée avec le laitier, sousproduit de la sidérurgie lorraine, de couleur grise et utilisée pour les bâtiments les plus communs (CAUE de la Meuse, 2007).

\section{La « bombturbation »}

C'est sur le site de Verdun que J. P. Hupy a réalisé sa thèse Assessing Landscape Disturbance and Recovery Across a WWI Battlefield : Verdun, France (2005), qui devait se poursuivre par sa publication avec Schaetzel (2006) définissant la bombturbation comme la formation d'entonnoirs à la surface du sol et le mélange des horizons pédologiques par des explosifs militaires, bombes, obus, mines. Ce type de pédoturbation d'origine humaine, connu dans d'autres zones de conflit (bombardements américains au Vietnam) a la particularité de pouvoir affecter des profondeurs plus grandes que les autres types de pédoturbation et d'aller plus profond que le sol, jusqu'à la roche-mère (Fig. 5).

Sur le front de l'Ouest, une bande de terrains dévastés s'étend sur une vingtaine de $\mathrm{km}$ de part et d'autre de la ligne de front, avec des zones plus perturbées que les autres et qu'on a définies comme "Zone rouge ». Sur le canton de Verdun, la dévastation a été particulièrement importante. Sur seulement $200 \mathrm{~km}^{2}$ sur lesquels l'artillerie allemande avait concentré une puissance de feu inouïe, on estime que les Allemands ont tiré 34 millions d'obus et les Français 26 millions de février à août 1916. Les obus percutants étaient particulièrement favorables à la perturbation du sol, une grande partie de l'énergie de l'explosion étant dirigée vers le sol, et un bombardement d'artillerie lourde pouvant pénétrer profondément au-dessous des horizons du sol, jusqu'à faire éclater la roche-mère.

La formation d'un cratère conduit à la disparition du profil pédologique en place. On ne retrouve plus que la roche-mère (ici calcaire) fracturée et les éjectas et débris de rocheassociés. La roche-mère et le sol, plusieurs fois retournés par la pluie d'obus, finissent par former une boue argileuse hétérogène, un peu comparable à une tillite de moraine glaciaire, surtout dans des zones où l'épaisseur de sol est faible.

Dans des cas extrêmes, le déplacement de matière a pu conduire à une disparition pure et simple d'un élément topographique. Ce fut le cas de la cote 304 sur la rive gauche de la Meuse, que les Allemands voulaient raser et qui perdit $6 \mathrm{~m}$ de calcaires tithoniens et kimméridgiens.

La perturbation du sol par les obus a aussi des impacts indirects sur l'environnement physique, qui peuvent avoir des conséquences sur l'évolution du sol, par exemple, des déplacements du plafond de la nappe phréatique locale. Dans quelques cas, la rochemère imperméable est perforée, et son drainage prive la végétation de son 
alimentation en eau, ou, à l'inverse, on peut observer des remontées d'une eau plus profonde générant des sols hydromorphes limitant les possibilités de reboisement ultérieur.

Ce phénomène a également été observé dans les Flandres, où la pénétration des argiles qui recouvraient des sables chargés d'eau sous pression par des obus de gros calibre faisait jaillir des eaux artésiennes qui remplissaient les trous d'obus. Elles s'ajoutaient aux pluies diluviennes pour contribuer à l'inondation du champ de bataille (Doyle, 2018). La nature argileuse et sableuse des formations des Flandres a fait qu'il n'a pas été conservé de trace de cette bombturbation.

Les effets de ces obus sont toujours visibles dans le paysage de Verdun, attestant de l'ampleur de la perturbation et de sa permanence. En surface, les pentes douces qui favorisaient l'écoulement superficiel sont à présent marquées de cratères de profondeurs diverses, qui ont une influence majeure sur l'hydrologie et le développement des sols qui en résulte. Le fond des cratères a recueilli une accumulation de matière organique, le socle rocheux frais a été altéré et certains de ses sous-produits lessivés. L'action de l'eau et la bioturbation par des organismes divers ont rapidement conditionné le développement d'un nouveau sol dans les fonds de cratère et ont conduit à une pédogenèse ultérieure peut-être plus rapide que ce qui serait arrivé autrement. Dans des zones où il y a une nappe perchée, comme la nappe du «Séquanien » qui alimentait les villages disparus du Quadrilatère des Forts, ou dans le fond des vallées, beaucoup de fonds de cratères sont en dessous de la surface piézométrique pour une partie significative de l'année. L'humidité empêche la reprise de l'évolution d'avant-guerre et introduit une nouvelle pédogenèse (sols hydromorphes à gley). On assiste à l'apparition de mares qui n'existaient pas avant.

Des études sur la pédoturbation liée au conflit sont actuellement en cours dans le saillant de Saint-Mihiel, à Sécheprey et à Flirey (Poszwa et al., 2018). Les terrains sont un peu plus anciens (marnes du Bathonien et calcaires du Bajocien), mais les observations réalisées sur un entonnoir concordent pour partie avec les effets d'une bombturbation. En effet, si à Verdun le terme d'éjecta est utilisé par Hupy et Schaetzl (2006) pour qualifier un mélange des horizons de sols avec de la roche, à Flirey l'éjecta est composé essentiellement de matériaux géologiques carbonatés durs. Les sols formés avant l'explosion et devant se trouver dans l'éjecta y sont tellement dilués qu'ils sont presque totalement indiscernables. Ils subsistent, en revanche, en place sous l'éjecta en bordure de cratère. Le terme de bombturbation à Flirey correspondrait donc à une modification de la morphologie avec la formation de cratères, la formation d'un éjecta et la fossilisation de l'ancien sol sous l'éjecta. L'effet bombturbation sur la pédogenèse post-conflit apparaît par contre très comparable à Flirey et à Verdun.

L'occupation humaine du territoire elle-même a été en grande partie réduite. Des villages ruraux qui, avant la bataille, formaient un réseau dense de structures d'habitation, ont purement et simplement disparu et sont réduits à des vestiges. De ces anciens villages, il ne reste souvent plus que quelques pierres et décombres entretenus pour la mémoire, et ce qui était autrefois des terres agricoles est maintenant recouvert par la forêt. À cet égard, Hupy et Schaetzl (2008) remarquent avec ironie que cette perturbation a permis au paysage de revenir au paysage boisé qu'il était avant la Révolution. Toutefois, la nature a eu besoin pour reprendre ses droits de l'intervention de l'office National des Forêts qui a mis fin à un processus d'embroussaillement des 
pelouses calcaires. Ces dernières sont régulièrement entretenues, ce qui a permis le maintien d'un milieu ouvert et l'installation de prairies remarquables. Ainsi, autour de l'ouvrage de Froideterre, la pauvreté du sol et l'ensoleillement ont créé un microclimat chaud et sec, propice au développement d'une faune et d'une flore thermophile, comprenant par exemple l'Orchis pyramidal (Anacamptispyramidalis), le criquet Oedipode turquoise(Oedipoda caerulescens) et le Machaon (Papilio machaon). L'eau retenue dans les trous d'obus forme un ensemble de petites mares (Fig. 6) où se sont développées une flore et une faune caractéristiques des zones humides: Grenouille rousse (Rana temporaria), Triton alpestre (Ichthyosaura alpestris), libellules comme l'Anax empereur (Anax imperator).

Document 6 : Ouvrage de Froideterre. Bombturbation et préservation de la biodiversité des mares dans les cratères par l'ONF (Cliché J.-C. Porchier)

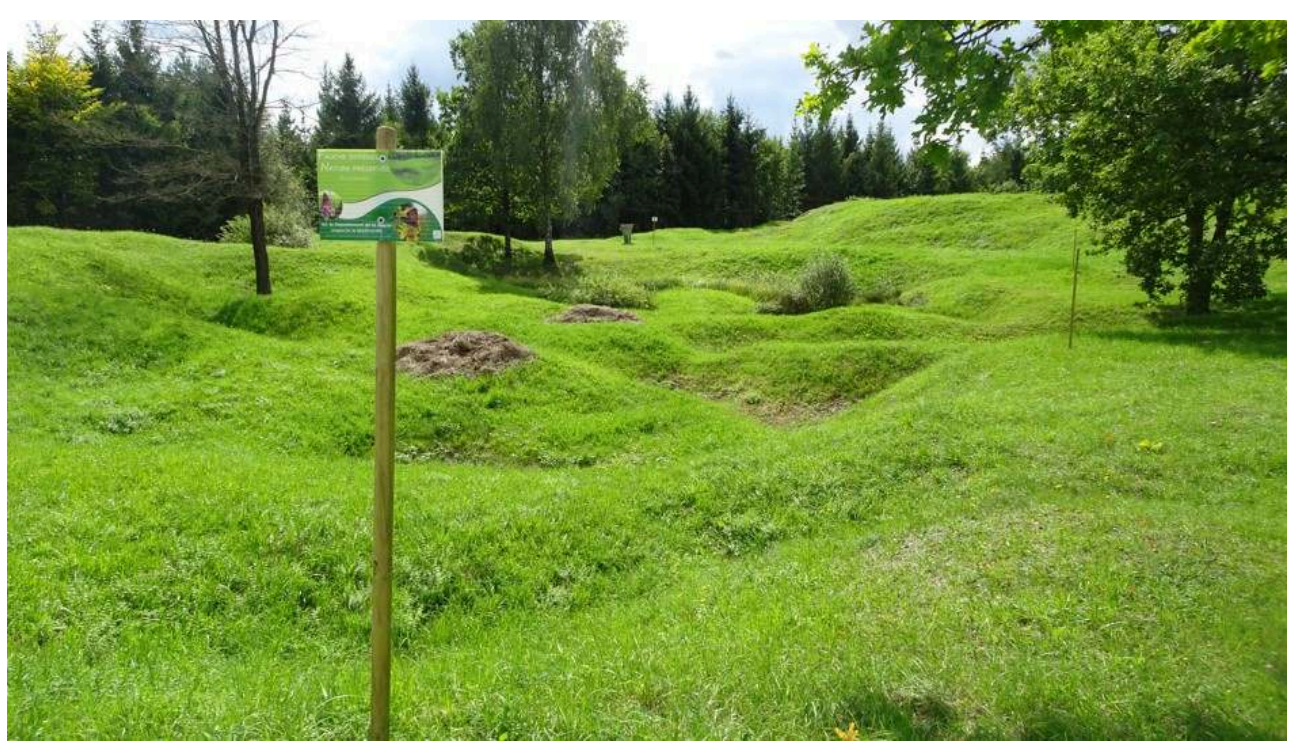

Dans le domaine botanique, on signalera également quelques plantes obsidionales, terme que les botanistes utilisent pour les végétaux qui ont été propagés lors des conflits armés ou des occupations militaires. Parmi les espèces de la région de Verdun, on relève le Panicaut géant (Eryngium giganteumL) apporté du Caucase par les Russes et la Bermudienne des montagnes ou Herbe aux yeux bleus (Sisyrinchium montanumGreene), apportée par les Américains.

\section{Conclusion}

Du fait de la protection que lui assuraient le front de côte et les ravins des Hauts de Meuse et de la présence au milieu du champ de bataille de la vallée de la Meuse qui empêchait la coordination de leurs offensives sur les deux rives, Verdun était difficile à prendre. Les Allemands ont dû appuyer leur attaque sur une concentration d'artillerie sans précédent dans l'histoire. Ces bombardements, auxquels a répondu l'artillerie française ont décapé les sols agricoles jusqu'à la roche ou les ont rendus impropres à la culture. La Meuse a ainsi perdu près de $20 \%$ de son territoire agricole, surtout dans le canton de Verdun. Cette destruction pyrotechnique, pour laquelle on a créé le nom de bombturbation, est masquée par le reboisement des terrains incultivables, dont le fait 
qu'il ait été réalisé en résineux au lieu des feuillus des forêts locales est le principal mais discret indice de leur ancienne occupation agricole.

Cette perturbation a également changé l'hydrologie des eaux de surface, les caractéristiques de la nappe phréatique et le processus d'évolution des sols ainsi que la vitesse de ce processus. Dans certains cas, ces changements, conjugués à une moindre intervention humaine ont accru la biodiversité et la richesse de la forêt.

La violence des combats et l'impossibilité de fixer le front ont empêché la construction d'ouvrages de terrain pérennes et la mise en place de systèmes de mines en dehors de ceux des deux extrémités, Vauquois au nord, Les Éparges au sud. Les réseaux de tranchées et de boyaux ne sont plus observables, même si l'emploi du lidar permet d'en révéler le tracé.

\section{BIBLIOGRAPHIE}

Académie de Nancy-Metz, 2016, Paysages et empreintes de la guerre, accédé le 16 août 2018, URL : http:// www4.ac-nancy-metz.fr/verdun2016/ paysages-etape3.html

Amat J-P., 2015, Les forêts dans la Grande Guerre : Histoire, mémoire, patrimoine

Cabouret M., 1989, « Etat actuel du remembrement en Lorraine. »In. : Revue Géographique de l'Est, Tome 29, $\mathrm{N}^{\circ} 3-4,1989$. Transport et nouvelles dimensions internationales. Perspectives européennes. pp. 313- 314 ; URL : https://www.persee. fr/doc/rgest_0035-3213_1989_num3_1710 ; DOI : https://doi. org/10.3406/rgest.1989.1710

CAUE de la Meuse, 2007, Reconstruction des années 1920 en Meuse : un patrimoine à protéger, CAUE de la Meuse (éd.), $41 \mathrm{p}$.

De Matos-Machado R., Amat J.-P., Arnaud-Fassetta G. et Bétard F., 2016, « Potentialités de l'outil LiDAR pour cartographier les vestiges de la Grande Guerre en milieu intra-forestier (bois des Caures, forêt domaniale de Verdun, Meuse) », EchoGéo [En ligne], 38 | 2016, mis en ligne le 13 décembre 2016, consulté le 12 août 2018. URL : http://journals. openedition.org/echogeo/14791 ; DOI : 10.4000/echogeo.14791

Doyle P., 2018, « Les Flandres belges : des belligérants aux prises avec l'eau et la boue. » In : 14-18, la Terre et le Feu. Géologie et géologues sur le front occidental, AGBP- COFRHIGÉO - SGN co-éd., Mém. hors-série $n^{\circ} 10$ de l'AGBP.

Harmand D., Porchier J.-C. et Le Roux J., 2018, « Verdun, un site emblématique », In. : 14-18, la Terre et le Feu. Géologie et géologues sur le front occidental, AGBP-COFRHIGÉO -SGN co-éd., Mém. horssérie nº 10 de l’AGBP, pp. 211- 225.

Hupy J., 2005, Assessing landscape disturbance and recovery across a WWI battlefield: Verdun, France. Thèse, East Lansing (Etats-Unis), Michigan State University, 209 p.

Hupy J. et Schaetzl R., 2006, « Introducing « bombturbation », a singular type of soil disturbance and mixing. » Soil science, 171, 11, pp. 823-836. DOI: 10.1097/01. ss.0000228053.08087.19 
Hupy J. et Schaetzl R., 2008, « Soil development on the WWI battlefield of Verdun, France » Geoderma, 145, pp. 37-49.

Hupy J. et Koehler T., 2012, « Modern warfare as a significant form of zoogeomorphic disturbance upon the landscape », Geomorphology, 157- 158, pp. 169-182

Ministère de l'Agriculture, 1936, Statistique agricole de la France, résultats généraux de l'enquête de 1929, Paris, Imprimerie nationale, 803 p.

Musset R., 1922, « La reconstitution agricole des régions dévastées du nord et du nord-est de la France. » In. : Annales de Géographie, t. 31, n¹72, 1922. pp. 355-360; doi : https://doi. org/10.3406/ geo.1922.10135; URL : https://www.persee.fr/doc/geo_0003- 4010_1922_num_31_172_10135

ONF, 2005, Aménagement de la Forêt domaniale de Verdun - 2006-2020, document ONF, 113 p., 3 cartes.

Poszwa A., Bartier D., Ollive V. et Moulis C., 2018, « Les impacts de la Grande Guerre sur l'évolution des paysages et des sols : l'exemple de Flirey, à l'est du saillant de Saint- Mihiel. » In. : 14-18, la Terre et le Feu. Géologie et géologues sur le front occidental, AGBP- COFRHIGÉO - SGN coéd., Mém. hors-série ${ }^{\circ} 10$ de l'AGBP.

Pranard C., 1842, Sedan Pittoresque, Ou Topographie, Statistique, Géographie, Histoire Et Biographie de L'Arrondissement de Sedan, 184 p., 1 carte.

Prudhomme A., 1893, Agriculture du département de la Meuse, Bar-le-Duc, Contant-Laguerre imprimeur-éditeur, 374 p., 1 carte h.t.

Solard, 1935, Fortification. Tome II : La fortification pendant la guerre 1914-1918. École d'Application d'Artillerie éd., Fontainebleau, 148 p., 7 planches h.t.

\section{RÉSUMÉS}

Au cœur du relief de côtes de la Lorraine sédimentaire, Verdun bénéficiait de solides défenses naturelles qui la protégeaient des attaques depuis l'est et le nord. À l'est, elle était protégée par la dépression marécageuse de la Woëvre, impropre aux manœuvres militaires, et par le front de la côte de Meuse. Au nord, la voie était barrée par des côtes et ravins SW-NW, découpant le plateau des Hauts de Meuse. La vallée de la Meuse, surimposée au relief avec ses méandres encaissés et son lit marécageux, a fait obstacle à la coordination des attaques allemandes sur les deux rives. L'impact de la bataille de Verdun sur le paysage se traduit moins par ce qu'on voit que parce qu'on ne voit plus. L'alternance d'attaques et de contre-attaques n'a pas permis l'installation d'ouvrages de terrain pérennes et la conservation des tranchées, et le reboisement de l'après-guerre a fait oublier qu'à la place de la forêt actuelle, il y avait un terroir agricole et forestier de type openfield, qui a perdu près de 100000 ha de terres labourable devenues incultivables. Les traces de la guerre se voient aussi dans la reconstruction des villages et des fermes entreprise dans les années 20. Enfin, la «bombturbation » et l'apparition de nouvelles espèces dans les espaces laissés à la nature témoignent également de l'impact de la bataille.

In the heart of the cuesta landscape of the sedimentary Lorraine, Verdun benefited from strong natural defences that protected the place from attacks from the east and the north. On the east side, it was protected by the marshy depression of the Woëvre, which was unsuitable for the movements of troops, and by the east-facing escarpment of the Meuse cuesta. On the north side, SW-NW ridges and ravines dissecting the plateau of the Hauts de Meuse were obstacles on the way of the German forces. The valley of the Meuse, overimposed on the plateau with its steep meanders and marshy bed, separated the two wings of the attacking armiesand prevented their 
efficient co-operation.The impact of the battle on the landscape is less reflected in what can be seen than in what cannot be seen any longer. Continuous attacks and counter-attacks did not allow the building of perennial defences and the conservation of trenches, and post-war reforestation made forget that where there is now a forest, there was an open-field system, which lost nearly 100,000 ha of arable land that had become uncultivable. The traces of war can also be seen in the reconstruction of villages and farms undertaken in the 1920s. Finally, the "bombturbation" and the emergence of new species in the spaces left to nature are also evidence of the impact of the battle.

\section{INDEX}

Mots-clés : Verdun, cuesta, Meuse, Oxfordien, fortifications, terroir, agriculture

Keywords : Verdun, cuesta, Meuse river, Oxfordian, fortifications, arable land, farming

\section{AUTEURS}

\section{JEAN-CLAUDE PORCHIER}

jeanclaude.porchier@ponts.org

DOMINIQUE HARMAND

dominique.harmand@univ-lorraine.fr

JACQUES LE ROUX

jacques.leroux@club-internet.fr 